\title{
Quantum kinetic equation in the rotating frame and chiral kinetic theory
}

\author{
Ömer F. Dayi and Eda Kilinçarslan \\ Physics Engineering Department, Faculty of Science and Letters, Istanbul Technical University, \\ TR-34469 Maslak-Istanbul, Turkey
}

(Received 17 July 2018; published 10 October 2018)

\begin{abstract}
A modified quantum kinetic equation which takes account of the noninertial features of rotating frame is proposed. The vector and axial-vector field components of the Wigner function for chiral fluids are worked out in a semiclassical scheme. It is demonstrated that the chiral currents and energy-momentum tensor computed by means of them are consistent with the hydrodynamical results. A new semiclassical covariant chiral transport equation is established by inspecting the equations satisfied by the chiral vector fields. It uniquely provides a new three-dimensional semiclassical chiral kinetic theory possessing a Coriolis force term. The particle number and current densities deduced from this transport equation satisfy the anomalous continuity equation and generate the magnetic and vortical effects correctly.
\end{abstract}

DOI: 10.1103/PhysRevD.98.081701

\section{INTRODUCTION}

Chiral fermions appear in different sorts of physical systems like heavy-ion collisions [1,2], electroweak plasma in early Universe [3-5] and Weyl semimetals in condensed matter physics [6-8]. In these systems collective dynamical properties are affected by chiral anomalies which yield the chiral magnetic effect $[1,2,9]$, the chiral separation effect $[10,11]$, the chiral vortical effect [12] and the local (spin) polarization effect [13-15]. The latter two are due to the rotation of reference frame. Chiral particles have been studied within the relativistic hydrodynamical approach in [12,16-18]. Dynamics of relativistic fluids can also be investigated by means of the quantum kinetic equation (QKE)

$$
\gamma_{\mu}\left(p^{\mu}+\frac{i \hbar}{2} \nabla^{\mu}\right) W(x, p)=0
$$

derived from the Dirac equation coupled to electromagnetic fields $[19,20]$, in the phase space described by the position and momentum four-vectors $x_{\mu}, p_{\mu}$. We consider a constant electromagnetic field strength $F_{\mu \nu}$, so that $\nabla^{\mu}=$ $\partial^{\mu}-Q F^{\mu \nu} \partial_{\nu}^{p}$, where $\partial^{\mu} \equiv \partial / \partial x_{\mu}, \partial_{\mu}^{p} \equiv \partial / \partial p^{\mu} . W(x, p)$ is the Wigner function for spin- $1 / 2$ fermions which can be decomposed in terms of sixteen independent generators of the Clifford algebra whose coefficients are scalar, pseudoscalar, vector, axial-vector and tensor fields. When fermions are massless the vector and axial-vector fields satisfy a set of

Published by the American Physical Society under the terms of the Creative Commons Attribution 4.0 International license. Further distribution of this work must maintain attribution to the author(s) and the published article's title, journal citation, and DOI. Funded by SCOAP. coupled equations. In [15] chiral vector fields were established semiclassically by considering the chiral fluid in the vicinity of local equilibrium. Thus, $x_{\mu}$ dependence of the fields is due to the fluid four-velocity $u_{\mu}(x)$. Then the derivatives of fields generate terms depending on the fluid vorticity $\omega^{\mu}=(1 / 2) \epsilon^{\mu \nu \alpha \rho} u_{\nu} \partial_{\alpha} u_{\rho}$.

QKE may also be employed to designate relativistic semiclassical chiral kinetic theory (CKT). Kinetic theory, needless to say, is essential to study the dynamics of chiral quark plasma. We consider only collisionless kinetic theories. There are mainly two different Lorentz covariant approaches which employ QKE: In [21] CKT was established by acting $\nabla_{\mu}$ on the vector and axial-vector fields derived in [15] where the momentum dependence of distribution function is only due to the component parallel to $u_{\mu}$, namely $u \cdot p$. The vorticity dependent terms acquired in [21] do not lead the desired currents and energy-momentum tensor. To surmount this difficulty one should add some terms to the time evolutions of phase space variables [22]. Moreover, in this method three-dimensional (3D) semiclassical CKT is not uniquely determined. The other relativistic approach $[23,24]$ is based on the chiral vector fields computed perturbatively within the quantum field theory. These can also be deduced from (1) by studying its solution in a frame moving with the velocity $n_{\mu}$ [25]. A covariant CKT is provided by acting $\nabla_{\mu}$ on them. Unfortunately, 3D limit of this relativistic equation for nonvanishing vorticity has not been discussed. Different from these, there is a worldline formalism approach to CKT [26].

In [27] it was claimed that the vorticity appearing in the hydrodynamical approach matches the angular velocity of the fluids in the comoving frame. In fact, they acquired the chiral vortical effect in 3D from the chiral magnetic effect by substituting magnetic field with angular velocity times energy suggested by the similarity of the Coriolis and 
Lorentz forces. This statement inspired the explicitly spatial coordinate dependent 3D semiclassical kinetic theory formulation of fermions presented in [28]. In this method the Coriolis force appears in contrary to the formulations of [21,24,25] based on QKE.

Here we follow a different route. The QKE derived from the Dirac equation including electromagnetic interactions is frame independent. In the absence of background fields it cannot yield a transport equation (TE) possessing terms which can be interpreted as force, so that noninertial forces like the Coriolis force do not appear. We propose a modification of (1) by making use of enthalpy current, such that the modified QKE suits well with the noninertial properties of the frame. Although, the modified QKE is valid for fermions either massive or massless, we only deal with chiral fluids. We first present the set of equations for chiral vector fields and discuss their semiclassical solutions by a general distribution function. To ensure the consistency of the modified QKE we present its solution for chiral vector fields in the comoving frame of fluid by choosing a specific distribution function and show that they lead to the correct vector, axial-vector currents and energy-momentum tensor. Then by employing the chiral vector fields written in terms of general distribution function, a covariant chiral transport equation (CTE) is established in the comoving frame of the fluid. Procedure of deriving the $3 \mathrm{D}$ limit of a four-dimensional TE by integrating it over $p_{0}$ is clarified and applied to the covariant CTE. We showed that a unique 3D CKT follows. The phase space measure, velocity and force imposed by this new CKT generate the desired continuity equations, magnetic effects, vortical effects as well as the Coriolis force. Our results demonstrate that the modified QKE is reliable and the proposed modification is essential to satisfactorily address the noninertial features of the rotating frame.

\section{MODIFIED QUANTUM KINETIC EQUATION}

The fluid four-velocity $u_{\mu}=d x_{\mu} / d \tau$, is defined by the proper time $\tau$, so that it satisfies $u_{\mu} u^{\mu}=1$. The metric tensor is $g_{\mu \nu}=\operatorname{diag}(1,-1,-1,-1)$. One can split a fourvector $y_{\mu}$ in directions parallel and perpendicular to $u_{\mu}$ as $y_{\mu}=y_{0} u_{\mu}+\bar{y}_{\mu}$, where $y_{0} \equiv y \cdot u$ and $\bar{y}_{\mu} \equiv\left(g_{\mu \nu}-u_{\mu} u_{\nu}\right) y^{\nu}$. The (non)inertial properties of relativistic vorticity are provided by the circulation tensor [29]

$$
W_{\mu \nu}=\partial_{\mu} W_{\nu}-\partial_{\nu} W_{\mu},
$$

where $W_{\mu}=h u_{\mu}$ is the enthalpy current given in terms of the internal energy (enthalpy) $h=p \cdot u$. For fluids which are not subject to acceleration $a_{\mu}=u_{\nu} \partial^{\nu} u_{\mu}=0$, the circulation tensor can be expressed as

$$
W_{\mu \nu}=2 h \Omega_{\mu \nu}+\left(\partial_{\mu} h\right) u_{\nu}-\left(\partial_{\nu} h\right) u_{\mu},
$$

where $\Omega_{\mu \nu}=\frac{1}{2}\left(\partial_{\mu} u_{\nu}-\partial_{\nu} u_{\mu}\right)$ is the (kinematic) vorticity tensor. Observe that the force acting on a fluid element imposed by (1) is characterized by the coefficient of the derivative with respect to momentum four-vector and there is an apparent resemblance between $F_{\mu \nu}$ and $W_{\mu \nu}$. Hence, to take into account the noninertial forces one is tempted to modify (1) by adding a term proportional to $W^{\mu \nu} \partial_{\nu}^{p}$ to $\nabla^{\mu}$. However in the rest frame of massive particles where $h$ is constant, the Coriolis force should vanish. Therefore, we add $\left(W^{\mu \nu}-2 h \Omega^{\mu \nu}\right) \partial_{\nu}^{p}$ to $\nabla^{\mu}$ to take into account the noninertial forces. Generalizing this consideration to a frame moving with the four-velocity $n_{\mu}$, satisfying $n_{\mu} n^{\mu}=1$, we define

$$
\tilde{\nabla}_{(n)}^{\mu} \equiv \partial_{x}^{\mu}-\left[Q F^{\mu \nu}+\left(\partial^{\mu} n^{\alpha}\right) p_{\alpha} n^{\nu}-\left(\partial^{\nu} n^{\alpha}\right) p_{\alpha} n^{\mu}\right] \partial_{\nu}^{p} .
$$

Therefore, for a constant field strength $F^{\mu \nu}$ we propose

$$
\gamma_{\mu}\left(p^{\mu}+\frac{i \hbar}{2} \tilde{\nabla}_{(n)}^{\mu}\right) W(x, p)=0,
$$

as the QKE in the rotating frame of reference.

There are similarities between the proposed modification and shifting gauge fields [30,31] to deal with moving medium. Our approach resembles the effective field theory formalism given in [30] where the gauge field is shifted by the fluid velocity multiplied by chemical potential. However, treating the enthalpy current as a gauge field does not yield the desired modification. Even if one can introduce a gauge field like function to generate the modification, it should be proportional to internal energy which depends on momentum. This would create problems in Lagrange formulation defined in terms of spacetime coordinates and derivatives with respect to them.

We are interested in the chiral vector fields

$$
\mathcal{J}_{\chi}^{\mu}=\frac{1}{2}\left(\mathcal{V}^{\mu}+\chi \mathcal{A}^{\mu}\right)
$$

constructed by the vector and axial-vector field components $\mathcal{V}_{\mu}$ and $\mathcal{A}_{\mu}$ of $W(x, p)$. They correspond to the right-handed $\chi=1$, and left-handed $\chi=-1$, fermions. The modified QKE (2), yields the following set of equations

$$
\begin{gathered}
p_{\mu} \mathcal{J}_{\chi}^{\mu}=0 \\
\tilde{\nabla}_{(n)}^{\mu} \mathcal{J}_{\chi \mu}=0 \\
\hbar \epsilon_{\mu \nu \alpha \rho} \tilde{\nabla}_{(n)}^{\alpha} \mathcal{J}_{\chi}^{\rho}=-2 \chi\left(p_{\mu} \mathcal{J}_{\chi \nu}-p_{\nu} \mathcal{J}_{\chi \mu}\right),
\end{gathered}
$$

which are decoupled from the other components.

\section{SEMICLASSICAL APPROXIMATION}

To study solutions of (3)-(5) we expand $\mathcal{J}_{\chi}^{\mu}$ in $\hbar$ and keep the zeroth- and first-order terms [15]: $\mathcal{J}_{\chi}^{\mu}=$ $\mathcal{J}_{\chi}^{(0) \mu}+\hbar \mathcal{J}_{\chi}^{(1) \mu}$. The zeroth-order solution of (3) and (5) is 


$$
\mathcal{J}_{\chi}^{(0) \mu}=p^{\mu} \delta\left(p^{2}\right) f_{\chi}^{0}
$$

$f_{\chi}^{0}$ is a general distribution function which can be decomposed into the particle and antiparticle parts, $s= \pm 1$, as $f_{\chi}^{0}=\sum_{s= \pm 1} \theta(s n \cdot p) f_{s, \chi}^{0}(x, p)$.

At the $\hbar$-order (3) and (5) lead to

$$
\begin{gathered}
p^{\mu} \mathcal{J}_{\chi \mu}^{(1)}=0, \\
\epsilon^{\mu \nu \alpha \rho} \tilde{\nabla}_{(n) \alpha} \mathcal{J}_{\chi \rho}^{(0)}=-2 \chi\left[p^{\mu} \mathcal{J}_{\chi}^{(1) \nu}-p^{\nu} \mathcal{J}_{\chi}^{(1) \mu}\right] .
\end{gathered}
$$

The general form of $\mathcal{J}_{\chi \mu}^{(1)}$ satisfying (7) and (8) is

$$
\begin{aligned}
\mathcal{J}_{\chi}^{(1) \mu}= & p^{\mu} f_{\chi}^{1} \delta\left(p^{2}\right)+\frac{1}{2} \chi Q \epsilon^{\mu \nu \alpha \beta} F_{\alpha \beta} p_{\nu} f_{\chi}^{0} \delta^{\prime}\left(p^{2}\right) \\
& +\chi \epsilon^{\mu \nu \alpha \rho} p_{\nu}\left(\partial_{\alpha} n_{\beta}\right) p^{\beta} n_{\rho} f_{\chi}^{0} \delta^{\prime}\left(p^{2}\right)+\mathcal{K}^{\mu},
\end{aligned}
$$

where $\delta^{\prime}\left(p^{2}\right)=-\delta\left(p^{2}\right) / p^{2}$ and $f_{\chi}^{1}$ is a general function which can be considered as the first-order part of the distribution function: $f_{\chi} \equiv f_{\chi}^{0}+\hbar f_{\chi}^{1}$. Inspired by the results of [23-25] we obtain $\mathcal{K}^{\mu}$ satisfying (7) and (8) as

$$
\mathcal{K}^{\mu}=S_{(n)}^{\mu \nu}\left(\tilde{\nabla}_{(n) \nu} f_{\chi}^{0}\right) \delta\left(p^{2}\right)
$$

where

$$
S_{(n)}^{\mu \nu}=\frac{\chi}{2 n \cdot p} \epsilon^{\mu \nu \rho \sigma} p_{\rho} n_{\sigma}
$$

corresponds to spin. Distribution function should be chosen consistently to satisfy the remaining equation (4). This will lead to the definition of covariant semiclassical chiral equation. However, let us first demonstrate that there exists a solution of (3)-(5) yielding results which are consistent with the ones existing in the literature.

\section{FERMI-DIRAC DISTRIBUTION}

We would like to present a specific solution in the comoving frame of the fluid $n_{\mu}=u_{\mu}$, by choosing the distribution function $f_{\chi}^{0}$ as in [15]:

$$
f_{\chi}^{\mathrm{FD}}=\frac{2}{(2 \pi \hbar)^{3}} \sum_{s= \pm 1} \frac{\theta(s n \cdot p)}{e^{s\left(u \cdot p-\mu_{\chi}\right) / T}+1} .
$$

The chemical potentials of chiral particles $\mu_{\chi}$, are given in terms of the total and chiral chemical potentials as $\mu_{R, L}=\mu \pm \mu_{5}$. Note that one ignores the derivatives of theta functions which yield vanishing contribution when one performs four-momentum integrals to calculate physical quantities [15]. $\mathcal{J}_{\chi}^{\mathrm{FD}(0) \mu}=p^{\mu} \delta\left(p^{2}\right) f_{\chi}^{\mathrm{FD}}$ satisfies (4) for constant temperature $T$, by letting $\partial_{\alpha} \mu=-Q E_{\alpha}, \quad \partial_{\alpha} \mu_{5}=0, \quad \partial_{\mu} u_{\nu}=-\partial_{\nu} u_{\mu}$.

$E_{\mu}=F_{\mu \nu} u^{\nu}$ is the electric field. The magnetic field is $B_{\mu}=(1 / 2) \epsilon_{\mu \nu \alpha \rho} u^{\nu} F^{\alpha \rho}$. By substituting $f_{\chi}$ with (10) in (9) we get

$$
\begin{aligned}
\mathcal{J}_{\chi}^{\mathrm{FD} \mu}= & {\left[p^{\mu} \delta\left(p^{2}\right)+\hbar \chi Q p^{\nu}\left(u_{\nu} B^{\mu}-B_{\nu} u^{\mu}\right) \delta^{\prime}\left(p^{2}\right)\right.} \\
& +\hbar \chi p \cdot u p^{\nu}\left(u_{\nu} \omega^{\mu}-\omega_{\nu} u^{\mu}\right) \delta^{\prime}\left(p^{2}\right)+\hbar \chi(\omega \cdot p) p^{\mu} \delta^{\prime}\left(p^{2}\right) \\
& \left.+\hbar \chi \omega^{\mu} \delta\left(p^{2}\right)+\hbar \chi Q \epsilon^{\mu \nu \alpha \beta} p_{\nu} E_{\alpha} u_{\beta} \delta^{\prime}\left(p^{2}\right)\right] f_{\chi}^{\mathrm{FD}}
\end{aligned}
$$

The vorticity tensor is expressed in terms of the fluid vorticity $\omega^{\mu}$ as $\Omega^{\mu \nu}=\epsilon^{\mu \nu \alpha \rho} u_{\alpha} \omega_{\rho}$. One can explicitly show that $\left(\tilde{\nabla}^{\mu} \equiv \tilde{\nabla}_{(u)}^{\mu}\right)$

$$
\tilde{\nabla}^{\mu} \mathcal{J}_{\chi \mu}^{\mathrm{FD}}=0
$$

is satisfied. We made use of (11) and $\partial^{\mu} \tilde{F}_{\mu \nu}=0$ where $\tilde{F}_{\mu \nu}=(1 / 2) \epsilon_{\mu \nu \alpha \rho} F^{\alpha \rho}$, which can be expressed as $\tilde{F}_{\mu \nu}=$ $B_{\mu} u_{\nu}-B_{\nu} u_{\mu}+\epsilon_{\mu \nu \alpha \beta} E_{\alpha} u_{\beta}$.

Although the electric field and magnetic field dependent terms of (12) are the same with the ones obtained in [15], the vorticity dependent terms are different as it would be expected. However, both should yield the same currents and energy momentum tensor. We will demonstrate their equivalence by calculating the currents and energy-momentum tensor resulting from the solution (12).

The vector and axial-vector currents are defined by

$$
j^{\mu}=\int d^{4} p \mathcal{V}^{\mu}, \quad j_{5}^{\mu}=\int d^{4} p \mathcal{A}^{\mu} .
$$

By substituting $\mathcal{V}^{\mu}$ and $\mathcal{A}^{\mu}$ with

$$
\mathcal{V}^{\mu}=\sum_{\chi} \mathcal{J}_{\chi}^{\mathrm{FD} \mu}, \quad \mathcal{A}^{\mu}=\sum_{\chi} \chi \mathcal{J}_{\chi}^{\mathrm{FD} \mu},
$$

we obtain

$$
\begin{gathered}
j^{\mu}=n u^{\mu}+\xi_{B} B^{\mu}+\xi \omega^{\mu}, \\
j_{5}^{\mu}=n_{5} u^{\mu}+\xi_{B 5} B^{\mu}+\xi_{5} \omega^{\mu} .
\end{gathered}
$$

The total and axial number densities are acquired as

$$
\begin{aligned}
n & =\frac{\mu}{3 \pi^{2} \hbar^{3}}\left(\mu^{2}+3 \mu_{5}^{2}+\pi^{2} T^{2}\right), \\
n_{5} & =\frac{\mu_{5}}{3 \pi^{2} \hbar^{3}}\left(3 \mu^{2}+\mu_{5}^{2}+\pi^{2} T^{2}\right) .
\end{aligned}
$$

The chiral magnetic and chiral separation effects are given, respectively, by the second terms of (15) and (16), where the coefficients are calculated as 


$$
\xi_{B}=\frac{Q \mu_{5}}{2 \pi^{2} \hbar^{2}}, \quad \xi_{B 5}=\frac{Q \mu}{2 \pi^{2} \hbar^{2}} .
$$

Similarly, vorticity yields the chiral vortical and local polarization effects generated by the last terms in (15) and (16), with

$$
\xi=\frac{\mu \mu_{5}}{\pi^{2} \hbar^{2}}, \quad \xi_{5}=\frac{T^{2}}{6 \hbar^{2}}+\frac{\mu^{2}+\mu_{5}^{2}}{2 \pi^{2} \hbar^{2}} .
$$

The energy-momentum tensor $T^{\mu \nu}$ is defined through the vector field $\mathcal{V}^{\mu}$ and four-momentum $p^{\mu}$ as

$$
T^{\mu \nu}=\frac{1}{2} \int d^{4} p\left(p^{\mu} \mathcal{V}^{\nu}+p^{\nu} \mathcal{V}^{\mu}\right) .
$$

By substituting $\mathcal{V}^{\mu}$ with (14) and performing the momentum integrals one acquires

$$
\begin{aligned}
T^{\mu \nu}= & \varepsilon u^{\mu} u^{\nu}+P\left(u^{\mu} u^{\nu}-g^{\mu \nu}\right)+n_{5}\left(u^{\mu} \omega^{\nu}+u^{\nu} \omega^{\mu}\right) \\
& +\frac{Q \xi}{2}\left(u^{\mu} B^{\nu}+u^{\nu} B^{\mu}\right) .
\end{aligned}
$$

Pressure is $P=\varepsilon / 3$ and the energy density is computed as

$$
\varepsilon=\frac{1}{2 \pi^{2}}\left(\frac{7 \pi^{2} T^{4}}{30}+\pi^{2} T^{2}\left(\mu^{2}+\mu_{5}^{2}\right)+3 \mu^{2} \mu_{5}^{2}-\frac{\mu^{4}+\mu_{5}^{4}}{2}\right) .
$$

By employing (11) one can show that the continuity equations are consistent with the chiral anomaly $\partial_{\mu} j^{\mu}=0$, $\partial_{\mu} j_{5}^{\mu}=-\left(Q^{2} / 2 \pi^{2}\right) E \cdot B$. Moreover, energy-momentum conservation can be established as $\partial_{\mu} T^{\mu \nu}=Q F^{\nu \alpha} j_{\alpha}$.

All the results obtained by employing the solution (12) coincide with the ones presented in [15]. Therefore we can conclude that the modified chiral QKE is consistent with the results established in relativistic hydrodynamics.

\section{CHIRAL TRANSPORT EQUATIONS}

We can now proceed to formulate the covariant semiclassical CTE from (6) and (9), by working in the comoving frame of fluid by setting $n_{\mu}=u_{\mu}$. Chiral vector fields are defined by the set of equations (3)-(5). However, (6) and (9) satisfy only two of them. The remaining equation (4), yields

$$
\begin{aligned}
\tilde{\nabla}_{\mu} \mathcal{J}_{\chi}^{\mu}= & \delta\left(p^{2}+\hbar \chi Q \frac{u_{\mu} \tilde{F}^{\mu \nu} p_{\nu}}{u \cdot p}\right)\{p \cdot \tilde{\nabla} \\
& +\frac{\hbar \chi Q}{u \cdot p} S^{\mu \nu} E_{\mu} \tilde{\nabla}_{\nu}-\frac{\hbar \chi}{u \cdot p} p_{\mu} \tilde{\Omega}^{\mu \nu} \tilde{\nabla}_{\nu} \\
& \left.+\frac{\hbar \chi}{u \cdot p}\left(\tilde{\Omega}^{\mu \nu} p_{\mu} u_{\nu}\right) \Omega^{\sigma \rho} p_{\rho} \partial_{\sigma}^{(p)}\right\} f_{\chi}=0 .
\end{aligned}
$$

We defined $\tilde{\Omega}_{\mu \nu}=\frac{1}{2} \epsilon_{\mu \nu \alpha \rho} \Omega^{\alpha \rho}$ and $S^{\mu \nu} \equiv S_{(u)}^{\mu \nu}$. Observe that in (9) there is a freedom in choosing $f_{\chi}^{1}$, which permits us to redefine it as

$$
f_{\chi}^{1} \Rightarrow \chi \frac{S^{\mu \nu} \Omega_{\mu \nu}}{u \cdot p} f_{\chi}^{0}+f_{\chi}^{1}
$$

The first term is similar to the rotation dependent part of the frame independent equilibrium distribution function given in [32]. Although this choice is essential to acquire a welldefined 3D limit, it does not violate the uniqueness of the 3D limit. A simple dimensional analysis show that another term which is linear in vorticity and $f_{\chi}^{0}$ does not exist. Therefore we define the covariant CTE as

$$
\begin{aligned}
& \delta\left(p^{2}+\hbar \chi Q \frac{u_{\mu} \tilde{F}^{\mu \nu} p_{\nu}}{u \cdot p}\right)\left\{p \cdot \tilde{\nabla}\left(1+\hbar \chi \frac{S^{\mu \nu} \Omega_{\mu \nu}}{u \cdot p}\right)\right. \\
& \quad+\frac{\hbar \chi Q}{u \cdot p} S^{\mu \nu} E_{\mu} \tilde{\nabla}_{\nu}-\frac{\hbar \chi}{u \cdot p} p_{\mu} \tilde{\Omega}^{\mu \nu} \tilde{\nabla}_{\nu} \\
& \left.\quad+\frac{\hbar \chi}{u \cdot p}\left(\tilde{\Omega}^{\mu \nu} p_{\mu} u_{\nu}\right) \Omega^{\sigma \rho} p_{\rho} \partial_{\sigma}^{(p)}\right\} f_{\chi}=0 .
\end{aligned}
$$

To ensure its covariance (frame independence) the distribution function $f_{\chi}=f_{\chi}^{0}+\hbar f_{\chi}^{1}$ should be chosen to transform appropriately [23-25,32]. The main difference between the CTE obtained in [24,25] and (17) lies in the vorticity dependent terms. Obviously another dissimilarity is the fact that (17) is defined in the comoving frame of fluid in contrary to the CTE of [24,25] which was claimed to be valid in any frame with velocity $n_{\mu}$. The electromagnetic field dependent terms and the mass-shell condition coincide by substituting $\tilde{\nabla}_{\mu}$ with $\nabla_{\mu}$.

A method of obtaining the 3D TE which is correlative to a four-dimensional TE is to integrate the latter over $p_{0}$ $[33,34]$. However, integrating four-dimensional TE naively over $p_{0}$ does not always yield a well-defined 3D equation. This goal can be achieved if the coefficients of $\partial f_{\chi} / \partial p^{\mu}$ integrated over $p_{0}$ permit writing

$$
\begin{aligned}
& \dot{\boldsymbol{p}}\left[\frac{\partial f_{\chi}(x, p)}{\partial \boldsymbol{p}}\right]_{p_{0}=\mathcal{E}}+\dot{\boldsymbol{p}} \cdot \frac{\partial \mathcal{E}}{\partial \boldsymbol{p}}\left[\frac{\partial f_{\chi}(x, p)}{\partial p_{0}}\right]_{p_{0}=\mathcal{E}} \\
& =\dot{\boldsymbol{p}} \frac{\partial f_{\chi}(t, \boldsymbol{x}, \mathcal{E}, \boldsymbol{p})}{\partial \boldsymbol{p}}
\end{aligned}
$$

where $\mathcal{E}$ will be dictated by the delta function of the relativistic equation. It is worth noting that physical quantities are defined in terms of integrals over momentum variables both in four- and three-dimensions, so that it is sufficient to relate the transport equations as

$$
\int d^{4} p\{4 \mathrm{D} \mathrm{TE}\}=\int d^{3} p\{3 \mathrm{D} \mathrm{TE}\} .
$$

We will integrate (17) over $p_{0}$ in the frame: $u^{\mu}=(1, \mathbf{0})$ and $\omega^{\mu}=(0, \boldsymbol{\omega})$. It is possible to satisfy (18) by setting 


$$
\begin{aligned}
\int d^{4} p \delta\left(p^{2}\right)\left\{E \cdot \omega\left(\frac{f_{\chi}^{0}}{p_{0}}-\frac{1}{2} \frac{\partial f_{\chi}^{0}}{\partial p_{0}}\right)\right. \\
\left.+\frac{\omega \cdot p E \cdot p}{p_{0}^{2}}\left(\frac{2 f_{\chi}^{0}}{p_{0}}-\frac{1}{2} \frac{\partial f_{\chi}^{0}}{\partial p_{0}}\right)\right\}=0 .
\end{aligned}
$$

One can show that this condition is fulfilled for $f_{\chi}^{0}=f_{\chi}^{\mathrm{FD}}$, which is the natural choice for fermions. Then we can conclude that $\int d^{4} p\{(17)\}=\int d^{3} p\{3 \mathrm{D} \mathrm{CTE}\}=0$, where vanishing of the latter integrand leads to the following $3 \mathrm{D}$ CTE,

$$
\left.(\sqrt{\eta})_{s}^{\chi} \frac{\partial}{\partial t}+(\sqrt{\eta} \dot{\boldsymbol{x}})_{s}^{\chi} \cdot \frac{\partial}{\partial \boldsymbol{x}}+(\sqrt{\eta} \dot{\boldsymbol{p}})_{s}^{\chi} \cdot \frac{\partial}{\partial \boldsymbol{p}}\right) f_{\chi, s}^{\mathrm{eq}}(t, \boldsymbol{x}, \boldsymbol{p})=0,
$$

with

$$
\begin{aligned}
\sqrt{\eta}_{s}^{\chi}=1 & +\hbar s Q \chi \boldsymbol{b}_{s} \cdot \boldsymbol{B}, \\
(\sqrt{\eta} \dot{\boldsymbol{x}})_{s}^{\chi}= & \boldsymbol{v}_{s}^{\chi}+\hbar \chi\left(\hat{\boldsymbol{p}} \cdot \boldsymbol{b}_{s}\right)\left(s Q \boldsymbol{B}+2 \mathcal{E}_{s}^{\chi} \boldsymbol{\omega}\right) \\
& +\hbar s Q \chi \boldsymbol{E} \times \boldsymbol{b}_{s}-2 \hbar \chi\left(\boldsymbol{\omega} \cdot \boldsymbol{b}_{s}\right) \boldsymbol{p}, \\
(\sqrt{\eta} \dot{\boldsymbol{p}})_{s}^{\chi}= & s Q \boldsymbol{E}+s Q \boldsymbol{v}_{s}^{\chi} \times \boldsymbol{B}+\boldsymbol{v}_{s}^{\chi} \times \mathcal{E}_{s}^{\chi} \boldsymbol{\omega} \\
& +\hbar \chi Q^{2} \boldsymbol{b}_{s}(\boldsymbol{E} \cdot \boldsymbol{B})-2 \hbar \chi\left(\boldsymbol{\omega} \cdot \boldsymbol{b}_{s}\right) \boldsymbol{p} \times \mathcal{E}_{s}^{\chi} \boldsymbol{\omega} \\
& -2 \hbar s Q \chi\left(\boldsymbol{\omega} \cdot \boldsymbol{b}_{s}\right) \boldsymbol{p} \times \boldsymbol{B} .
\end{aligned}
$$

We introduced the Berry curvature $\boldsymbol{b}_{s}=\boldsymbol{s} \boldsymbol{p} / 2|\boldsymbol{p}|^{3}$. Dispersion relation and the canonical velocity are

$$
\begin{aligned}
\mathcal{E}_{s}^{\chi} & =|\boldsymbol{p}|\left(1-\hbar s Q \chi \boldsymbol{b}_{s} \cdot \boldsymbol{B}\right), \\
\boldsymbol{v}_{s}^{\chi} & =\frac{\partial \mathcal{E}_{s}^{\chi}}{\partial \boldsymbol{p}}=\hat{\boldsymbol{p}}\left(1+2 \hbar s Q_{\chi} \boldsymbol{b}_{s} \cdot \boldsymbol{B}\right)-\hbar s Q \chi b_{s} \boldsymbol{B} .
\end{aligned}
$$

The third term of (21) is the Coriolis force. It is one half of the force that one naively expects to find by substituting mass with $\mathcal{E}_{s}^{\chi}$ in the classical Coriolis force of a massive particle.

The 3D formalism of [28] is different from (19)-(22). One of the main differences is in the dispersion relation: (22) does not possess a vorticity dependent term in contrary to the one given in $[27,28]$. Although the vorticity dependent dispersion relation can be generated by shifting $\boldsymbol{p}$ in (22), it is an open question if the current formalism and the one given in [28] are related by a phase space coordinate transformation as it is discussed in [35].

By making use of (19) and (20) we can define the chiral particle (antiparticle) number and current densities as

$$
n_{s}^{\chi}=\int[d p](\sqrt{\eta})_{s}^{\chi} f_{\chi}^{\mathrm{eq}, s},
$$

$$
\dot{j}_{s}^{\chi}=\int[d p](\sqrt{\eta} \dot{\boldsymbol{x}})_{s}^{\chi} f_{\chi}^{\mathrm{eq}, s}+\boldsymbol{\nabla} \times \int[d p] \mathcal{E}_{s}^{\chi} \boldsymbol{b}_{s}^{\chi} f_{\chi}^{\mathrm{eq}, s}
$$

where $[d p]=d^{3} p /(2 \pi \hbar)^{3}$. One can easily observe that they satisfy the continuity equation

$$
\frac{\partial n_{s}^{\chi}}{\partial t}+\boldsymbol{\nabla} \cdot \dot{j}_{s}^{\chi}=\left.\frac{\chi Q^{2}}{(2 \pi \hbar)^{2}} \boldsymbol{E} \cdot \boldsymbol{B} f_{\chi}^{\mathrm{eq}, s}\right|_{p=0}
$$

The equilibrium partition function of a rotating fluid [32] in the frame which we integrated (17) is

$$
f_{\chi}^{\mathrm{eq}, s}=\frac{1}{e^{\left(\mathcal{E}_{s}^{\chi}-s \mu_{\chi}-\hbar s \lambda \hat{p} \cdot \boldsymbol{\omega} / 2\right) / T}+1} .
$$

By employing it one can show that the current (24) yields the chiral magnetic and the chiral separation effects $\boldsymbol{j}_{V}^{\mathrm{CME}}=\xi_{B} \boldsymbol{B}, \boldsymbol{j}_{A}^{\mathrm{CSE}}=\xi_{B 5} \boldsymbol{B}$, the chiral vortical and the local (spin) polarization effects $\boldsymbol{j}_{V}^{\mathrm{CVE}}=\xi \boldsymbol{\omega}, \boldsymbol{j}_{A}^{\mathrm{LPE}}=\xi_{5} \boldsymbol{\omega}$. They are consistent with (15) and (16).

\section{CONCLUSIONS}

QKE for fermions is modified to take into consideration the noninertial properties of the rotating coordinate frame. We have constructed its solution for chiral fermions by choosing the distribution function as Fermi-Dirac and calculated the vector, axial-vector currents and energy momentum tensor. We showed that they are consistent with the hydrodynamical approach which is a necessary condition for being an acceptable theory. We defined a new 4D CTE. We established its 3D limit which is shown to yield correct anomalous effects as well as continuity equation for number and current densities. It is a new CKT which is not explicitly coordinate dependent and accommodates the Coriolis force. Various physical systems can be studied within these covariant and 3D transport equations. For example they are extremely useful in investigating the nonlinear transport properties of chiral plasma (see [36] and the references therein). Incorporating collisions into the covariant CKT can be addressed by the methods developed in $[23,24,32]$. An important aspect of the modified QKE which is left to future studies is to explore its field theoretical origins.

\section{ACKNOWLEDGMENTS}

This work is supported by the Scientific and Technological Research Council of Turkey (TÜBİTAK) Grant No. 117F328. 
[1] D. E. Kharzeev, L. D. McLerran, and H. J. Warringa, The effects of topological charge change in heavy ion collisions: Event by event P and CP violation, Nucl. Phys. A803, 227 (2008).

[2] K. Fukushima, D. E. Kharzeev, and H. J. Warringa, Chiral magnetic effect, Phys. Rev. D 78, 074033 (2008).

[3] M. Joyce and M. E. Shaposhnikov, Primordial Magnetic Fields, Right Electrons, and the Abelian Anomaly, Phys. Rev. Lett. 79, 1193 (1997).

[4] H. Tashiro, T. Vachaspati, and A. Vilenkin, Chiral effects and cosmic magnetic fields, Phys. Rev. D 86, 105033 (2012).

[5] J. R. Bhatt and A. K. Pandey, Primordial magnetic field and kinetic theory with Berry curvature, Phys. Rev. D 94, 043536 (2016).

[6] X. Wan, A. M. Turner, A. Vishwanath, and S. Y. Savrasov, Topological semimetal and Fermi-arc surface states in the electronic structure of pyrochlore iridates, Phys. Rev. B 83, 205101 (2011).

[7] A. A. Burkov and L. Balents, Weyl Semimetal in a Topological Insulator Multilayer, Phys. Rev. Lett. 107, 127205 (2011).

[8] K.-Y. Yang, Y.-M. Lu, and Y. Ran, Quantum Hall effects in a Weyl semimetal: Possible application in pyrochlore iridates, Phys. Rev. B 84, 075129 (2011).

[9] D. Kharzeev and A. Zhitnitsky, Charge separation induced by P-odd bubbles in QCD matter, Nucl. Phys. A797, 67 (2007).

[10] M. A. Metlitski and A. R. Zhitnitsky, Anomalous axion interactions and topological currents in dense matter, Phys. Rev. D 72, 045011 (2005).

[11] K. Jensen, P. Kovtun, and A. Ritz, Chiral conductivities and effective field theory, J. High Energy Phys. 10 (2013) 186.

[12] D. T. Son and P. Surówka, Hydrodynamics with Triangle Anomalies, Phys. Rev. Lett. 103, 191601 (2009).

[13] Z. T. Liang and X. N. Wang, Globally Polarized QuarkGluon Plasma in Noncentral A + A Collisions, Phys. Rev. Lett. 94, 102301 (2005).

[14] F. Becattini, F. Piccinini, and J. Rizzo, Angular momentum conservation in heavy ion collisions at very high energy, Phys. Rev. C 77, 024906 (2008).

[15] J.-H. Gao, Z.-T. Liang, S. Pu, Q. Wang, and X.-N. Wang, Chiral Anomaly and Local Polarization Effect from the Quantum Kinetic Approach, Phys. Rev. Lett. 109, 232301 (2012).

[16] M. V. Isachenkov and A. V. Sadofyev, The chiral magnetic effect in hydrodynamical approach, Phys. Lett. B 697, 404 (2011).

[17] D. E. Kharzeev and H.-U. Yee, Anomalies and time reversal invariance in relativistic hydrodynamics: The second order and higher dimensional formulations, Phys. Rev. D 84, 045025 (2011).

[18] Y. Neiman and Y. Oz, Relativistic hydrodynamics with general anomalous charges, J. High Energy Phys. 03 (2011) 023 .
[19] H. T. Elze, M. Gyulassy, and D. Vasak, Transport equations for the QCD quark Wigner operator, Nucl. Phys. B276, 706 (1986).

[20] D. Vasak, M. Gyulassy, and H. T. Elze, Quantum transport theory for Abelian plasmas, Ann. Phys. (N.Y.) 173, 462 (1987).

[21] J.-W. Chen, S. Pu, Q. Wang, and X.-N. Wang, Berry Curvature and Four-Dimensional Monopoles in the Relativistic Chiral Kinetic Equation, Phys. Rev. Lett. 110, 262301 (2013).

[22] J.-H. Gao, S. Pu, and Q. Wang, Covariant chiral kinetic equation in Wigner function approach, Phys. Rev. D 96, 016002 (2017).

[23] C. Y. Hidaka, S. Pu, and D. L. Yang, Relativistic chiral kinetic theory from quantum field theories, Phys. Rev. D 95, 091901 (2017).

[24] C. Y. Hidaka, S. Pu, and D. L. Yang, Nonlinear responses of chiral fluids from kinetic theory, Phys. Rev. D 97, 016004 (2018).

[25] A. Huang, S. Shi, Y. Jiang, J. Liao, and P. Zhuang, Complete and Consistent Chiral Transport from Wigner Function Formalism, Phys. Rev. D 98, 036010 (2018).

[26] N. Mueller and R. Venugopalan, Worldline construction of a covariant chiral kinetic theory, Phys. Rev. D 96, 016023 (2017).

[27] M. A. Stephanov and Y. Yin, Chiral Kinetic Theory, Phys. Rev. Lett. 109, 162001 (2012).

[28] Ö. F. Dayi, E. Kilinçarslan, and E. Yunt, Semiclassical dynamics of Dirac and Weyl particles in rotating coordinates, Phys. Rev. D 95, 085005 (2017).

[29] L. Rezzola and O. Zanotti, Relativistic Hydrodynamics (Oxford University Press, New York, 2013).

[30] A. V. Sadofyev, V. I. Shevchenko, and V. I. Zakharov, Notes on chiral hydrodynamics within the effective theory approach, Phys. Rev. D 83, 105025 (2011).

[31] N. Banerjee, J. Bhattacharya, S. Bhattacharyya, S. Jain, S. Minwallaa, and T. Sharma, Constraints on fluid dynamics from equilibrium partition functions, J. High Energy Phys. 09 (2012) 046.

[32] B. J.-Y. Chen, D. T. Son, and M. A. Stephanov, Collisions in Chiral Kinetic Theory, Phys. Rev. Lett. 115, 021601 (2015).

[33] P. Zhuang and U. Heinz, Relativistic quantum transport theory for electrodynamics, Ann. Phys. (N.Y.) 245, 311 (1996).

[34] P. Zhuang and U. Heinz, Wigner functions in covariant and single-time formulations, Ann. Phys. (N.Y.) 266, 351 (1998).

[35] X. Huang and A. V. Sadofyev, Chiral vortical effect for an arbitrary spin, arxiv:1805.08779.

[36] Ö. F. Dayi and E. Kilinçarslan, Nonlinear chiral plasma transport in rotating coordinates, Phys. Rev. D 96, 043514 (2017). 